University of Nebraska - Lincoln

DigitalCommons@University of Nebraska - Lincoln

$5-26-2006$

\title{
Dynamics of ferroelectric domain growth in the field of atomic force microscope
}

A. Agronin

Tel Aviv University, Ramat-Aviv, Israel

M. Molotskii

Tel Aviv University, Ramat-Aviv, Israel

Y. Rosenwaks

Tel Aviv University, Ramat-Aviv, Israel

G. Rosenman

Tel Aviv University, Ramat-Aviv, Israel

\section{B. J. Rodriguez}

North Carolina State University, Raleigh, brian.rodriguez@ucd.ie

See next page for additional authors

Follow this and additional works at: https://digitalcommons.unl.edu/physicsgruverman

Part of the Physics Commons

Agronin, A.; Molotskii, M.; Rosenwaks, Y.; Rosenman, G.; Rodriguez, B. J.; Kingon, A. I.; and Gruverman, Alexei, "Dynamics of ferroelectric domain growth in the field of atomic force microscope" (2006). Alexei Gruverman Publications. 30.

https://digitalcommons.unl.edu/physicsgruverman/30

This Article is brought to you for free and open access by the Research Papers in Physics and Astronomy at DigitalCommons@University of Nebraska - Lincoln. It has been accepted for inclusion in Alexei Gruverman Publications by an authorized administrator of DigitalCommons@University of Nebraska - Lincoln. 


\section{Authors}

A. Agronin, M. Molotskii, Y. Rosenwaks, G. Rosenman, B. J. Rodriguez, A. I. Kingon, and Alexei Gruverman 


\title{
Dynamics of ferroelectric domain growth in the field of atomic force microscope
}

\author{
A. Agronin, ${ }^{\text {a) }}$ M. Molotskii, ${ }^{\text {b) }}$ Y. Rosenwaks, and G. Rosenman \\ Department of Electrical Engineering-Physical Electronics, School of Engineering, Tel Aviv University, \\ Ramat-Aviv 69978, Israel

\begin{abstract}
B. J. Rodriguez, A. I. Kingon, and A. Gruverman
Departments of Physics and Materials Science and Engineering, North Carolina State University, Raleigh,
\end{abstract} \\ North Carolina 27695
}

(Received 6 November 2005; accepted 20 March 2006; published online 26 May 2006)

\begin{abstract}
Application of very high voltage to atomic force microscope tip leads to the growth of narrow, stringlike domains in some ferroelectrics, a phenomenon that was named "ferroelectric domain breakdown." In this work the dynamics of domain breakdown have been studied experimentally and theoretically in stoichiometric lithium niobate (LN). The theory has been found to be in a good agreement with the measured domain radius temporal dependence. Dynamics of domain growth has also been studied in ultrathin LN crystals, where the domain breakdown phenomenon does not take place. It is also shown that domain formation processes occurring in bulk and ultrathin crystals are very different, and this is ascribed to the observed difference in depolarization energy dependence on the domain length. (C) 2006 American Institute of Physics. [DOI: 10.1063/1.2197264]
\end{abstract}

\section{INTRODUCTION}

During the last decade, there was a great interest in studies of micro- and nanosized ferroelectric domains generated and imaged by means of atomic force microscopy (AFM). ${ }^{1}$ This interest arises mainly from the potential application of nanodomain superlattices for a generation of electronic and photonic devices. Experiments show that evolution of domains tailored in thin films ${ }^{2-5}$ and bulk ferroelectrics ${ }^{6-11}$ by the use of AFM is very different. Application of a high bias of more than kilovolt to the tip led to the observation of the so-called "domain breakdown" in bulk ferroelectric crystals. ${ }^{8,9}$ This phenomenon is characterized by a formation of stringlike domains that continue to grow through the crystal thickness even though the tip-generated electric field reaches very low value at a large distance from the surface. In contrast, the domain breakdown cannot evolve in films or ultrathin crystals. In order to understand the effect of the boundary conditions on the mechanism of domain formation in the inhomogeneous field of AFM, one should perform a comparative analysis of domain growth kinetics in crystals with different thicknesses. Recently, detailed studies of the equilibrium domain shape dependence on the applied voltage were performed by us under the conditions of domain breakdown in congruent lithium niobate (LN) crystals. ${ }^{8,9,11}$

The analysis of domain growth dynamics in congruent crystals becomes significantly harder due to the existence of strong obstacles which pin the motion of domain walls (DW). ${ }^{12}$ The process of the DW overcoming the obstacles has not been studied yet which causes considerable difficulties in the interpretation of experiments on DW growth dynamics in ferroelectric congruent crystals. Therefore the DW dynamics should be studied in stoichiometric crystals, where

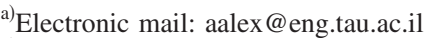

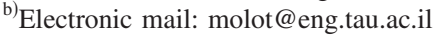

the defect and pinning center concentrations are very small. Domain growth dynamics was studied recently in ultrathin (0.85 $\mu \mathrm{m}$ thick) LN crystals of stoichiometric composition. ${ }^{13}$

In this work we perform experimental studies of domain breakdown kinetics in bulk stoichiometric LN crystals, and further develop the theory of domain growth kinetics for bulk and ultrathin crystals. We show that this theory can quantitatively explain our experimental data and the observed large difference in domain growth process between bulk and ultrathin ferroelectric crystals.

\section{EXPERIMENT}

We used optically polished Z-cut stoichiometric $0.5 \mathrm{~mm}$ thick $\mathrm{LiNbO}_{3}$ single crystals supplied by Oxide Corporation, Japan. The sample was glued to a bottom electrode using a conducting silver paste, and mounted onto the HVAFM scanner (Autoprobe CP, Veeco, Inc.), which was specifically modified for the high voltage application. ${ }^{14}$ Cantilever with spring constant $k=17 \mathrm{~N} / \mathrm{m}$ was used for domain writing and imaging. High voltage pulses of durations between $100 \mu \mathrm{s}$ and $1800 \mathrm{~s}$ of $U=1 \mathrm{kV}$ were applied between the AFM tip and the bottom electrode. Following each voltage pulse, a new domain was formed and subsequently imaged using piezoresponse force microscopy. ${ }^{15}$ Studies of the domain growth kinetics in ultrathin crystals were implemented by the use of $0.85 \mu \mathrm{m}$ thick stoichiometric $\mathrm{LiNbO}_{3}$ samples. The details of these experiments can be found in Ref. 13.

Figure 1 represents the experimental data (dotted line) averaged over all measurements for the same voltage pulse durations while the error bar represents the standard deviation. The solid curve in Fig. 1 represents the solution to the system of differential equations [(3a) and (3b)] presented in Sec. IV for the domain radius. In these calculations the DW mobility was a free parameter used to adjust the theory to the experimental data (solid curve in Fig. 1). The curve in Fig. 2 


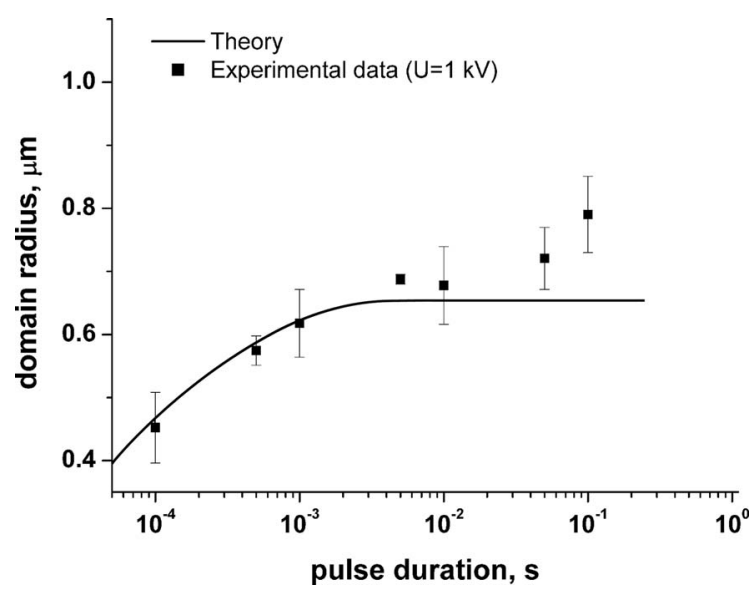

FIG. 1. Domain radius in bulk LN crystal vs the pulse duration for the applied voltage of $1 \mathrm{kV}$.

shows the domain elongation as a function of time obtained from the solution of the same set of equations [(3a) and (3b)]. The dots in Figs. 4-6 represent the experimental data obtained in Ref. 13, where the solid curves follow the dependencies expressed in Eqs. (9), (13), and (16) developed in Sec. V.

\section{DOMAIN WALL MOTION IN DYNAMICAL AND THERMAL FLUCTUATIONAL REGIMES}

The DW motion in a ferroelectric material requires overcoming of energy barriers related to periodic crystal structure (Miller-Weinreich barrier) and lattice defects. The slowly moving DW overcomes them by thermal fluctuations. In such a case, the DW velocity $v_{\text {DW }}$ is an exponential function of the electric field $E{ }^{16}$

$$
v_{\mathrm{DW}}(E)=v_{\infty} \exp \left(-\frac{\delta_{E}}{E}\right),
$$

where $\delta_{E}$ is an activation field, which decreases with temperature, and $v_{\infty}$ is a parameter having the dimensionality of velocity.

For high wall velocities, when the DW kinetic energy exceeds the energy barrier, a nonactivated type of motion

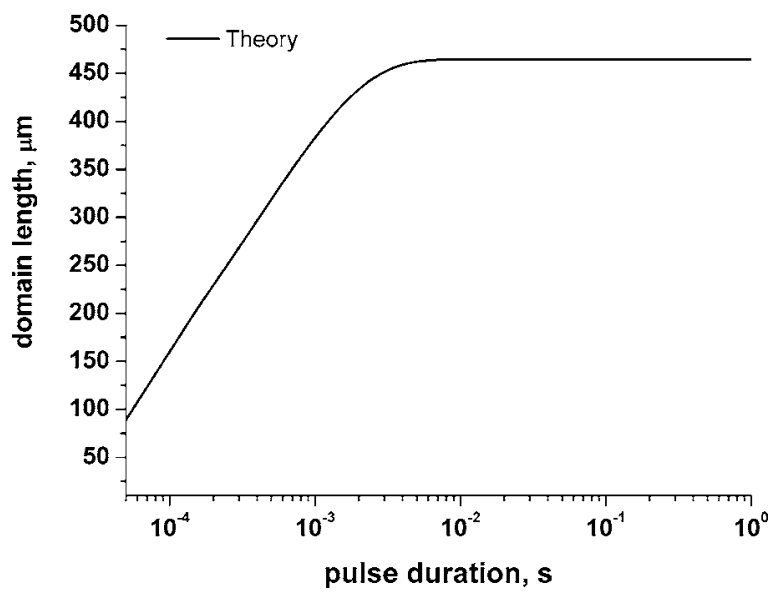

FIG. 2. Domain length in bulk $\mathrm{LN}$ vs time for the applied voltage of $1 \mathrm{kV}$. takes place. DW motion is limited by energy transfer from the DW to diverse elementary crystal excitations-mainly to phonons. In such a case, the DW motion is described by a viscous friction model and $v_{\mathrm{DW}}$ increases proportionally to the field, ${ }^{16}$

$$
v_{\mathrm{DW}}=\mu_{S} E
$$

where $\mu_{S}$ is the DW mobility.

Equation (2) replaces the dependence (1) for DW velocities larger than a characteristic velocity $v_{\mathrm{ch}}$. To date, there are no data on $v_{\text {ch }}$ in stoichiometric lithium niobate crystals. However, measurements conducted in congruent LN crystals ${ }^{17}$ yielded a value $v_{\mathrm{ch}} \sim 0.1 \mathrm{~cm} / \mathrm{s}$. It can be expected that in stoichiometric ferroelectric samples lacking strong pinning centers, the critical value of the velocity $v_{\mathrm{ch}}$ is smaller. Previous results ${ }^{13}$ showed that in AFM-induced switching in ultrathin LN crystal the DW velocity is smaller than $0.1 \mathrm{~cm} / \mathrm{s}$ for nearly entire range of domain lateral growth. On the other hand, under the conditions of domain breakdown in bulk crystal the wall velocity is higher than $v_{\mathrm{ch}}$ for the most of the growth process, and the velocity of the domain in the forward direction is still higher. Therefore we use (1) for domain growth analysis in ultrathin LN crystals, whereas Eq. (2) is used for the dynamic description of domain breakdown in the bulk samples. As will be shown below, the domain growth kinetics developed using these assumptions is in a good agreement with the experimental results in both cases.

\section{DOMAIN DYNAMICS IN THICK FERROELECTRIC CRYSTALS}

Ferroelectric domain reversal in the field of AFM consists of several stages ${ }^{18}$ which coincide with the stages of domain reversal in homogeneous fields. ${ }^{16}$ It starts from the nucleation of new domains with polarization vector pointing in the direction of the applied electric field. The activation energy of domain nucleation in very high field of AFM tip is negligibly small even for a small tip bias. ${ }^{18}$ Therefore, in contrast to the case of the uniform electric field, the nucleation stage under the AFM tip does not limit the domain reversal process. This fact allows us to neglect the nucleation stage while describing the domain growth dynamics.

As was already mentioned above, the fast motion of the domain walls in the case of domain breakdown can be described as a motion in a viscous medium. Loge and Suo have developed a theory describing such a motion, ${ }^{19}$ based on the assumption that the relation between DW velocity and the electric field is linear and can be described by Eq. (2). This theory was generalized in Ref. 18 for any dependence of DW velocity on the field and then used for the study of domain formation in the field of AFM.

When a domain radius is significantly larger then the tip apex curvature $R$ the field of the tip may be described within the so-called simple spherical model. ${ }^{20}$ In such a case the field of the AFM tip apex is supposed to coincide with a field of a single point charge located in a center of a metallic sphere of radius $R$. In general the total field of AFM tip can be found by superposition of two fields: the field of a charged cone and the field of a hemisphere located at the 
cone apex. ${ }^{21}$ The tip apex contributes mainly to the total electric field at the distances comparable to the radius of curvature of the hemisphere $R$. At larger distances from the tip apex, more significant contribution is provided by the cone. Measurements of domain radius as a function of the applied voltage $U$ between the tip and the bottom electrode showed ${ }^{11}$ that for $U<1.6 \mathrm{kV}$ this dependence can be well described by the theory ${ }^{9,10}$ where only tip apex contribution is taken into account. Calculations showed that adding the cone part destroys the agreement between theory and experiment. This unexpected result can be explained assuming that some other effect compensates the cone field, for example, ferroelectric indentation nearby the tip. ${ }^{7,22}$ The cone field leads to domain radius growth ${ }^{18}$ whereas indentation reduces it. ${ }^{7,22}$ Therefore we assume that in the range of voltages between $0<U<1.6 \mathrm{kV}$ these effects compensate each other. In the following discussion we will analyze domain breakdown dynamics for voltages smaller than $1.6 \mathrm{kV}$. Therefore the indentation and cone field contributions may be omitted and only the part of tip apex is taken into account.

We assume that under ferroelectric domain breakdown DWs move fast enough, so that the relation between velocity of DW and the electric field could be described by Eq. (2). Then, using the results obtained in Refs. 9, 10, and 18 we obtain the system of dynamics equations,

$$
\begin{aligned}
& \frac{d r}{d t}=\frac{3 \mu_{S}}{4 P_{S} \pi l^{2}}\left[f-\frac{1}{4}\left(\frac{17 c r^{3}}{l}+3 b l\right)\right], \\
& \frac{d l}{d t}=\frac{3 \mu_{S}}{16 \pi P_{S} r l}\left[\frac{1}{2}\left(\frac{11 c r^{3}}{l}-b l\right)-f\right],
\end{aligned}
$$

which describe the domain growth in the field of AFM. Here $r$ is the domain radius, $l$ is the domain length,

$$
f=\frac{8 \pi C_{\mathrm{ts}} U P_{S}}{\sqrt{\varepsilon_{c} \varepsilon_{a}}+1}
$$

is an effective force exerted by the charged tip on the domain, where $U$ is the applied voltage, $P_{S}$ is the magnitude of spontaneous polarization, $\varepsilon_{a}$ and $\varepsilon_{c}$ are dielectric permittivities along lateral and polar axes, respectively,

$$
C_{\mathrm{ts}}=R \sinh \alpha \sum_{n=0}^{\infty}\left(\frac{\sqrt{\varepsilon_{c} \varepsilon_{a}}-1}{\sqrt{\varepsilon_{c} \varepsilon_{a}}+1}\right)^{n} / \sinh (n+1) \alpha
$$

is the tip-sample capacitance, here $\alpha$ is determined by

$$
\cosh \alpha=1+\frac{\delta}{R}
$$

$\delta$ is the distance between the tip apex and the sample surface,

$$
b=\frac{\pi^{2}}{2} \sigma_{W}
$$

is proportional to DW energy density $\sigma_{W}$, and

$$
c=\frac{16 \pi^{2} P_{S}^{2}}{3 \varepsilon_{a}}\left[\ln \left(\frac{2 l}{r} \sqrt{\frac{\varepsilon_{a}}{\varepsilon_{c}}}\right)-1\right]
$$

depends weakly on the domain dimensions.

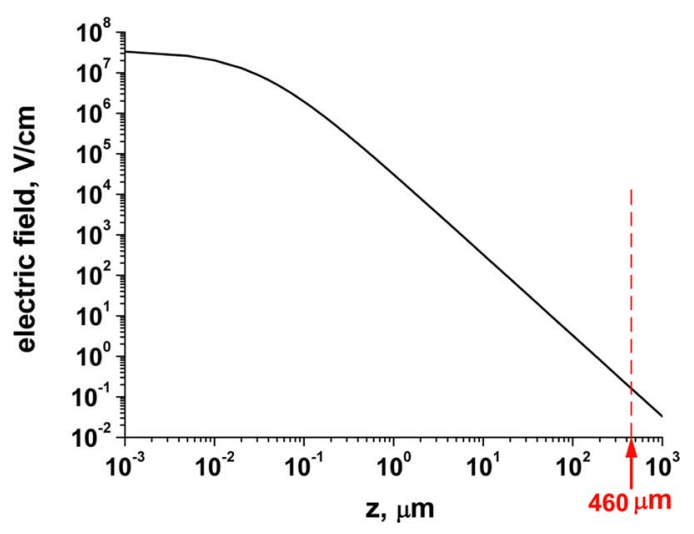

FIG. 3. (Color online) Calculated electric field in ferroelectric bulk LN crystals, tip radius of $50 \mathrm{~nm}$, and applied voltage of $1 \mathrm{kV}$. The arrow points to the equilibrium domain length obtained for $1 \mathrm{kV}$ as shown in Fig. 2.

The system of equations (3a) and (3b) was solved for a voltage $U=1 \mathrm{kV}$ and the following parameters: $\varepsilon_{c}=30$, $\varepsilon_{a}=84, P_{S}=75 \mu \mathrm{C} / \mathrm{cm}^{2}, R=50 \mathrm{~nm}, \delta=0.5 \mathrm{~nm}$, and $\sigma_{W}$ $=4.5 \mathrm{~mJ} / \mathrm{m}^{2}$. Figure 1 shows that the free parameter $\mu_{S}$ $=1.05 \times 10^{5} \mathrm{~mm}^{2} / \mathrm{kV} \mathrm{s}$, resulted in a good agreement of $r(t)$ with the experimental data (Fig. 1). The obtained value of DW mobility in stoichiometric LN crystals is five orders larger than the DW mobility value in congruent crystals obtained by Peng et al. ${ }^{17}$ This value is reasonable because it is close to the mobility value in other pure ferroelectrics with small defects concentration. For example Merz ${ }^{23}$ showed that in pure $\mathrm{BaTiO}_{3}$ the DW mobility $\mu_{S} \cong 2.5 \times 10^{5} \mathrm{~mm}^{2} / \mathrm{kV} \mathrm{s}$ is of the same order. In addition Fig. 1 shows that for this domain wall mobility, theory follows the experimental data for pulse durations shorter than $0.1 \mathrm{~s}$. For longer pulse durations there is a deviation from the proposed theory. A possible reason for this will be described in the Sec. V.

The electric field generated by the tip is very large on the crystal surface reaching $10^{8} \mathrm{~V} / \mathrm{cm}$ for high voltages necessary for domain breakdown. ${ }^{9}$ Figure 3 shows that the field decreases steeply farther away from the surface. At the distances comparable with the domain length (a few hundred of microns) this field does not exceed several tenth volts per centimeter. Therefore for long domains generated during the breakdown the contribution of the external field can be neglected. In our first work on ferroelectric domain breakdown ${ }^{9}$ it was shown that the field of the AFM tip does not lead directly to the domain elongation in the case of sufficiently long domains. It leads only to the domain radius growth, which causes increase in the internal force generated by the depolarization field and, as a result to the domain elongation.

\section{DOMAIN DYNAMICS IN ULTRATHIN FERROELECTRIC CRYSTALS}

The experimental studies of ultrathin ferroelectric stoichiometric crystals $\mathrm{LiNbO}_{3}$ were recently reported. ${ }^{13}$ Here we present a theory which describes this experimental data. It is shown below that the domain growth in ultrathin crystals can be explained using the same approach which was applied to the domain formation in thin films. ${ }^{24}$ We assume that the domain has a shape of a circular cylinder with radius $\rho$, and length $h$ equal to the crystal thickness. The total do- 
main energy still consists of the same three components as described in Sec. IV. However the energy terms are completely different. The surface energy $W_{S}$ equals to the product of surface energy density $\sigma_{W}$ and the surface area of the domain side $2 \pi \rho h$,

$$
W_{S}=2 \pi \rho h \sigma_{W} .
$$

The experimental results for ultrathin crystals ${ }^{13}$ were obtained in ambient environment in contrast to experiments on bulk crystals performed in dry nitrogen; this implies that the ultrathin crystal surface is covered by a water layer. ${ }^{2}$ In this case interaction energy between the AFM tip field and the domain becomes ${ }^{24}$

$$
W_{T}=-f_{\varepsilon} \rho,
$$

where

$$
f_{\varepsilon}=\frac{8 \pi P_{S} C_{\mathrm{ts}, \varepsilon} U}{\sqrt{\varepsilon_{c} \varepsilon_{a}}+\varepsilon_{w}}
$$

is the effective force applied to the domain in the case when the ferroelectric sample is located in an environment with dielectric permittivity $\varepsilon_{W}$. Then the tip-sample capacitance is $^{10,22}$

$$
C_{\mathrm{ts}, \varepsilon}=R \varepsilon_{w} \sinh \alpha \sum_{n=0}^{\infty}\left(\frac{\sqrt{\varepsilon_{c} \varepsilon_{a}}-\varepsilon_{w}}{\sqrt{\varepsilon_{c} \varepsilon_{a}}+\varepsilon_{w}}\right)^{n} / \sinh (n+1) \alpha,
$$

which is different from the corresponding expression for vacuum defined earlier.

The calculated equilibrium domain size is in agreement with the experimental data of Ref. 13 if we assume that the depolarization field is screened as a result of free charge redistribution inside the sample (internal screening), ${ }^{25}$ or as a result of ion adsorption on the surface (external screening). ${ }^{26}$ The depolarization energy can be written as follows: ${ }^{24}$

$$
W_{D}=\alpha_{S} \rho^{2} h,
$$

where

$$
\alpha_{s}=4 \pi^{2} P_{s}^{2} \beta^{2} \frac{\sqrt{\varepsilon_{c} \varepsilon_{a}}+1}{\sqrt{\varepsilon_{c} \varepsilon_{a}}}\left(\frac{1}{\sqrt{\varepsilon_{c} \varepsilon_{a}}+\varepsilon_{w}}+\frac{1}{\sqrt{\varepsilon_{c} \varepsilon_{a}}+\varepsilon_{m}}\right),
$$

$\beta$ is the degree of nonscreened part of $P_{S}$ and $\varepsilon_{m}$ is the dielectric permittivity of the bottom electrode. In the case of a metal top electrode $\left(\varepsilon_{m} \rightarrow \infty\right)$, and the second term in the summation may be neglected.

It should be emphasized here that depolarization energy for domain geometry in ultrathin crystals is proportional to the domain length, as shown by Eq. (7). This is in contrary to the depolarization energy in bulk crystals where it is inversely proportional to the domain length. Therefore, the main factor that influences domain dynamics is the ratio between the domain length and the crystal thickness. At the same time, the degree of screening cannot change the character of depolarization energy dependence on the domain length neither for the bulk nor for the ultrathin crystal.

Using Eqs. (4), (5), and (7), the total domain energy becomes

$$
W(\rho)=2 \pi \rho h \sigma_{W}-f_{\varepsilon} \rho+\alpha_{S} \rho^{2} h .
$$

The minimization of the energy in Eq. (8) makes it possible to obtain the equilibrium domain radius dependence on the applied tip voltage,

$$
\rho_{m}(U)=\frac{4 \pi P_{S} C_{\mathrm{ts}, \varepsilon}\left(U-U_{\mathrm{min}}\right)}{\left(\sqrt{\varepsilon_{c} \varepsilon_{a}}+\varepsilon_{w}\right) \alpha_{S} h}
$$

where

$$
U_{\min }=\frac{h \sigma_{W}\left(\sqrt{\varepsilon_{c} \varepsilon_{a}}+\varepsilon_{w}\right)}{4 P_{S} C_{\mathrm{ts}, \varepsilon}}
$$

is the threshold voltage necessary for the domain formation in the ultrathin crystal. The existence of a threshold voltage in thin films is a well known phenomenon. ${ }^{2-4,24}$ It is determined by minimal value of the pressure $f_{\varepsilon} / 2 \pi \rho h$, which causes a domain expansion in the field of AFM where it exceeds the Laplace pressure $\sigma_{W} / \rho$, that impedes such an expansion. Calculations ${ }^{7,18}$ show that the threshold exists also for domain formation in a bulk ferroelectric crystal. For $U>U_{\min }$ the domain radius depends linearly on voltage, which is consistent with the experimental data in Ref. 13. This behavior differs significantly from the $r_{m} \propto U^{2 / 3}$ dependence observed for equilibrium domains in bulk crystals. ${ }^{9-11}$ This difference originates from the different depolarization energy dependence on the domain size for these two cases.

The force acting on domain wall can be obtained from Eq. (8),

$$
F(\rho)=-\frac{\partial W(\rho)}{\partial \rho}=f_{\varepsilon}-2 \pi h \sigma_{W}-2 \alpha_{S} \rho h .
$$

In equilibrium $\rho=\rho_{m}$ and the force is zero leading to

$$
f_{\varepsilon}=2 \pi h \sigma_{W}+2 \alpha_{S} \rho_{m} h .
$$

The pressure applied on the DW is found according to

$$
p(\rho)=\frac{F(\rho)}{2 \pi \rho h}=\frac{\alpha_{S}\left(\rho_{m}-\rho\right)}{\pi \rho} .
$$

An electric field $E$ leads to a pressure $p$ that is equal to $2 P_{S} E$. Therefore, the equivalent field can be related to the pressure in Eq. (11) by

$$
E_{\mathrm{eq}}(\rho)=\frac{p(\rho)}{2 P_{S}}=\frac{\alpha_{S}\left(\rho_{m}-\rho\right)}{2 \pi P_{S} \rho} .
$$

Then, the wall velocity dependence on the domain radius can be obtained by substituting the equivalent field in Eq. (12) with the field $E$ in Eq. (1),

$$
v(\rho)=v_{\infty} \exp \left(-\frac{\eta \rho}{\rho_{m}-\rho}\right),
$$

where $\eta=2 \pi P_{S} \delta_{E} / \alpha_{S}$ and $v_{\infty}$ determines DW velocity at $\rho=0$ (under the AFM tip). Since the domain velocity grows with $U$, the value of $v_{\infty}$ will also grow with $U$. The simplest approximation for the $v_{\infty}$ dependence on $U$ in the range of voltages from 20 to $100 \mathrm{~V}$ is a linear function,

$$
v_{\infty}(U)=A+B\left(U-U_{0}\right),
$$

where $U_{0}=20 \mathrm{~V}$. 


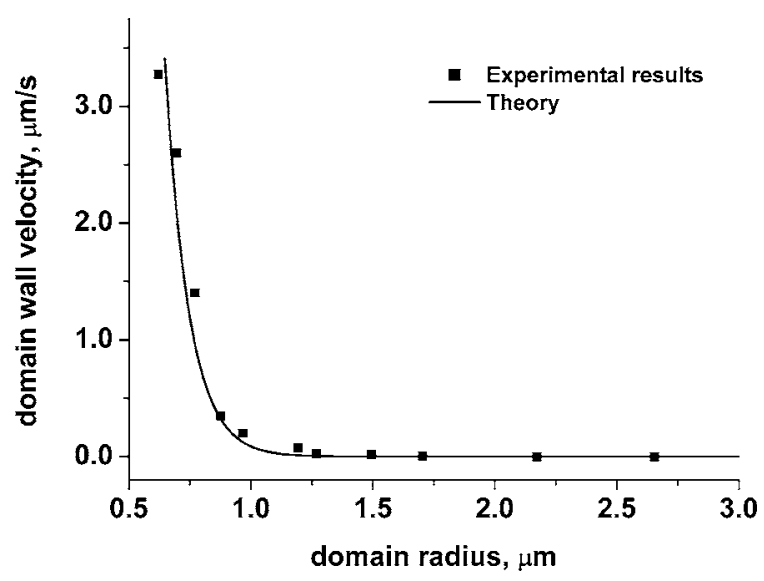

FIG. 4. Domain wall velocity in the ultrathin LN crystal vs domain radius for the pulse amplitude of $100 \mathrm{~V}$ (from Ref. 13) approximated by Eq. (13).

Equation (13) can be written as

$$
\frac{d \rho}{d t}=v_{\infty} \exp \left(-\frac{\eta \rho}{\rho_{m}-\rho}\right) \text {. }
$$

Here, Eq. (15) describes the domain growth kinetics in the field of AFM tip; the solution for $\rho \ll \rho_{m}$ is

$$
\rho(t)=\frac{\rho_{m}}{\eta} \ln \left(1+\frac{v_{\infty} \eta t}{\rho_{m}}\right) .
$$

Using expressions (9), (13), and (16) and the experimental results in Ref. 13 we obtain the following values of the parameters: $\delta_{E}=620 \mathrm{kV} / \mathrm{cm}, A=0.02 \mathrm{~cm} / \mathrm{s}, B=2.25$ $\times 10^{-3} \mathrm{~cm} / \mathrm{V} \mathrm{s}$, and $\beta=0.032$. The value of the unscreened portion of the depolarization field $\beta$ lies in the range of $0.01-$ 0.1 , as was obtained for other ferroelectrics. ${ }^{25}$ Figure 4 demonstrates a good agreement between theoretical and experimental data for the velocity dependence on a domain radius. The solid line in Fig. 5 shows the linear dependence of the domain radius on the applied voltage obtained according to Eq. (9). Domain equilibrium radius $\rho_{m}$ equals to 4,10 , and $20 \mu \mathrm{m}$ for voltages of 20,50 , and $100 \mathrm{~V}$, respectively. The calculation results agree with the experimental data in the

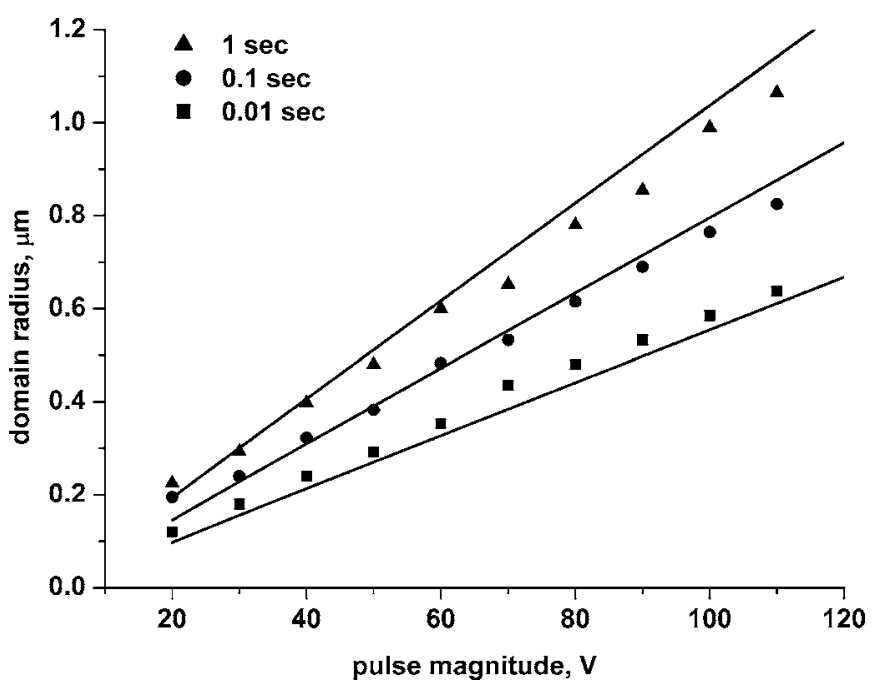

FIG. 5. Domain radius in the ultrathin LN crystal vs the pulse duration for various pulse magnitudes approximated by Eq. (9).

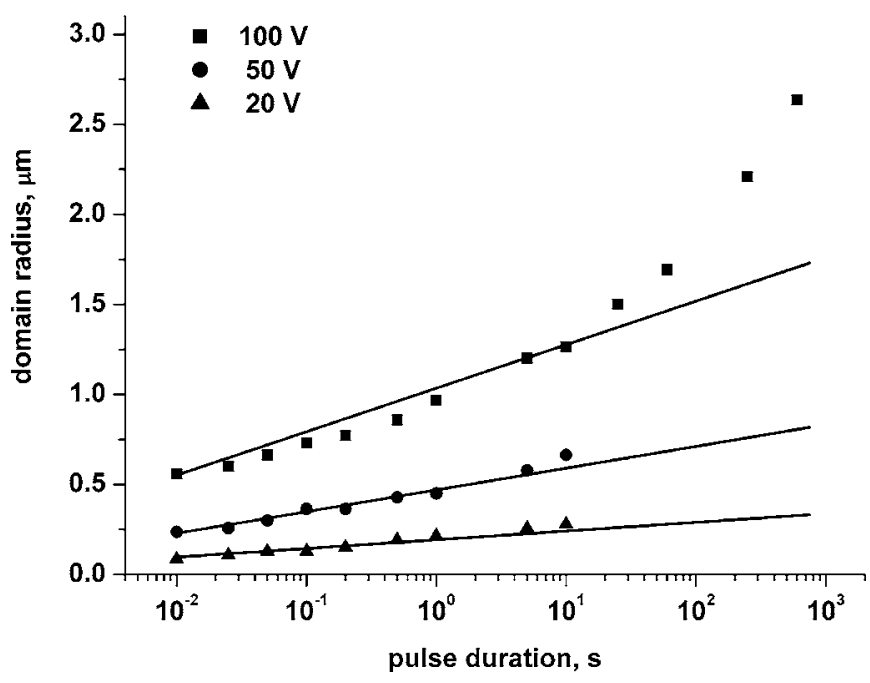

FIG. 6. Domain radius in ultrathin LN crystal vs pulse magnitude for three different pulse durations approximated by Eq. (16).

range of pulse durations from $10^{-2}$ to $\sim 30 \mathrm{~s}$ (Fig. 6). However, for longer pulses, domains are growing faster than it is predicted by Eq. (16) (Fig. 6); the same behavior is observed in bulk LN crystal. The reason for this deviation is still unclear and should be further studied. It might be a transformation of ferroelectric surface layer in the high field of the AFM tip in analogy to transformation of ionic crystals into superionic state under high fields. ${ }^{27}$ The fact that the theory follows the experimental data for high voltage experiment when shorter pulse durations are applied (Fig. 1) supports this assumption. According to our experiments where $U=1 \mathrm{kV}$ this pulse duration $(\sim 0.1 \mathrm{~s})$ is significantly shorter than for $U=20-100 \mathrm{~V}$ (more than $30 \mathrm{~s}$ ).

Let us estimate a threshold voltage $U_{\min }$. Using Eq. (6) the tip capacitance is calculated to be $C_{\mathrm{ts}, \varepsilon}=4 \times 10^{-16} \mathrm{~F}$ (for $R=50 \mathrm{~nm}, \delta=0.5 \mathrm{~nm}, \varepsilon_{c}=30, \varepsilon_{a}=84$, and $\left.\varepsilon_{w}=81\right)$. Then for $\sigma_{W}=4.5 \mathrm{~mJ} / \mathrm{m}^{2}, \quad \varepsilon_{m} \rightarrow \infty$, and $h=0.85 \mu \mathrm{m}$ we obtain $U_{\min }=0.04 \mathrm{~V}$. This value is negligible comparing to voltages of $20-100 \mathrm{~V}$ used in experiments on the ultrathin crystal. ${ }^{13}$ Therefore, in these experiments the threshold effect was not observed.

\section{CONCLUSIONS}

In this work the generalized theory of domain dynamics was developed to perform its quantitative comparison with our experiment and determine parameters of the theory. Such comparison provided very large DW mobility value for stoichiometric $\mathrm{LiNbO}_{3}$ which is close to the mobility of DW in pure $\mathrm{BaTiO}_{3}$ crystal. It is higher by five orders of magnitude than the value of mobility in congruent samples. The influence of an environment on the domain kinetics was taken into account. It was shown that domain equilibrium radius size dependence on the applied voltage differs significantly for ultrathin and bulk crystals. The growth kinetics is also different for both cases. In the beginning of the domain growth its radius increases steeply with time in bulk crystals, but it grows logarithmically slow when the domain approaches equilibrium state. On the other hand, the domain radius in ultrathin crystals always grows logarithmically in 
the studied range of voltages. It was shown that the observed qualitative difference in domain growth is mainly owing to the difference in the depolarization energy dependence on the domain size for these two cases.

\section{ACKNOWLEDGMENTS}

This work was supported by United States-Israel Binational Science Foundation, and Ministry of Science and Technology of Israel (Grant No. 637).

${ }^{1}$ Nanoscale Characterization of Ferroelectric Materials: Scanning Probe Microscopy Approach, edited by M. Alexe and A. Gruverman (Springer, Berlin, 2004)

${ }^{2}$ C. Durkan, M. E. Welland, D. P. Chu, and P. Migliorato, Phys. Rev. B 60 , 16198 (1999); Appl. Phys. Lett. 76, 366 (2000).

${ }^{3}$ P. Paruch, T. Tybell, and J. M. Triscone, Appl. Phys. Lett. 79, 530 (2001).

${ }^{4}$ T. Tybell, P. Paruch, T. Giamarchi, and J. M. Triscone, Phys. Rev. Lett. 89, 097601 (2002)

${ }^{5}$ C. S. Ganpule, A. L. Roytburd, V. Nagarajian, B. K. Hill, S. B. Ogale, E. D. Williams, R. Ramesh, and J. F. Scott, Phys. Rev. B 65, 014101 (2002)

${ }^{6}$ A. L. Gruverman, J. Hatano, and H. Tokumoto, Jpn. J. Appl. Phys., Part 1 36, 2207 (1997).

${ }^{7}$ M. Abplanalp, Ph.D. thesis, ETH Zürich, 2001; M. Abplanalp, J. Fousek, and P. Günter, Phys. Rev. Lett. 86, 5799 (2001).

${ }^{8}$ G. Rosenman, P. Urenski, A. Agronin, Y. Rosenwaks, and M. Molotskii, Appl. Phys. Lett. 82, 103 (2003).

${ }^{9}$ M. Molotskii, A. Agronin, P. Urenski, M. Shvebelman, G. Rosenman, and Y. Rosenwaks, Phys. Rev. Lett. 90, 107601 (2003).
${ }^{10}$ M. Molotskii, J. Appl. Phys. 93, 6234 (2003).

${ }^{11}$ A. Agronin, Y. Rosenwaks, and G. Rosenman, Appl. Phys. Lett. 85, 452 (2004).

${ }^{12}$ T. J. Yang, V. Gopalan, P. J. Swart, and U. Mohideen, Phys. Rev. Lett. 82, 4106 (1999); J. Phys. Chem. Solids 61, 275 (2000).

${ }^{13}$ B. J. Rodriguez, R. J. Nemanich, A. Kingon, A. Gruverman, S. V. Kalinin, K. Terabe, X. Y. Liu, and K. Kitamura, Appl. Phys. Lett. 86, 012906 (2005).

${ }^{14}$ G. Rosenman, Y. Rosenwaks, and P. Urenski, U.S. Patent No. 6,653,630 (25 2003 November).

${ }^{15}$ K. Franke, J. Besold, W. Haessler, and C. Seegebarth, Surf. Sci. Lett. 302, L283 (1994), A. Gruverman, O. Auciello, J. Hatano, and H. Tokumoto, Ferroelectrics 184, 11 (1996).

${ }^{16}$ F. Jona and G. Shirane, Ferroelectric Crystals (Pergamon, Oxford, 1962).

${ }^{17}$ L. H. Peng, Y. C. Fang, and Y. C. Lin, Appl. Phys. Lett. 74, 2070 (1999).

${ }^{18}$ M. Molotskii and M. Shvebelman, Philos. Mag. 85, 1637 (2005).

${ }^{19}$ R. E. Loge and Z. Suo, Acta Mater. 44, 3429 (1996).

${ }^{20}$ B. D. Terris, J. E. Stern, D. Rugal, and H. J. Mamin, Phys. Rev. Lett. 63, 2669 (1989); G. Zavala, J. H. Fendler, and S. Trolier-McKinstry, J. Appl. Phys. 81, 7480 (1997); K. Goto and K. Hane, ibid. 84, 4043 (1998).

${ }^{21}$ S. Hudlet, M. Saint Jean, C. Guthmann, and J. Berger, Eur. Phys. J. B B2, 5 (1998)

${ }^{22}$ S. V. Kalinin and D. A. Bonnell, Phys. Rev. B 65, 125408 (2002); S. V. Kalinin, E. Karapetian, and M. Kachanov, ibid. 70, 184101 (2004).

${ }^{23}$ W. J. Merz, Phys. Rev. 95, 690 (1954).

${ }^{24}$ M. Molotskii, J. Appl. Phys. 97, 014109 (2005).

${ }^{25}$ R. R. Mehta, B. D. Silverman, and J. T. Jacobs, J. Appl. Phys. 44, 3379 (1973); P. Würfel and I. P. Batra, Ferroelectrics 12, 55 (1976).

${ }^{26}$ G. Rosenman, D. Shur, Ya. E. Karasik, and A. Dunaevsky, J. Appl. Phys. 88, 6109 (2000)

${ }^{27}$ Yu. Ya. Gurevich and Yu. I. Kharkats, Phys. Rep. 139, 201 (1986). 